\title{
ESTUDO DE ALTERAÇÕES NA CAVIDADE ORAL EM PACIENTES COM DOENÇA DO REFLUXO GASTROESOFÁGICO
}

\author{
Maria Carolina Canteras Scarillo Falotico CORRÊA ${ }^{1}$, Mauro Masson LERCO² e \\ Maria Aparecida Coelho de Arruda HENRY2
}

\begin{abstract}
RESUMO - Racional - A doença do refluxo gastroesofágico, afecção de elevada e crescente incidência, pode se manifestar através de sintomas típicos (pirose e regurgitação) e atípicos (pulmonares, otorrinolaringológicos e bucais). Objetivo - Analisar as alterações na cavidade oral de pacientes com a doença do refluxo gastroesofágico. Métodos - Foram estudados 100 pacientes, sendo 50 acometidos por doença do refluxo gastroesofágico (grupo 1) e 50 controles (grupo 2). Todos os pacientes foram submetidos a exame clínico oral e questionário específico, e naqueles do grupo 1, foram realizadas endoscopia digestiva alta e manometria e pHmetria esofágicas. Resultados - A endoscopia digestiva alta demonstrou esofagite em todos os pacientes, sendo erosiva em 20, não-erosiva em 30 e hérnia hiatal em 38 . A pressão média no esfíncter inferior do esôfago foi de $11 \pm 4,8 \mathrm{~mm} \mathrm{Hg}$ e no superior de $75 \pm 26,5 \mathrm{~mm} \mathrm{Hg}$. Em 42 pacientes do grupo 1 (84\%) foi observado refluxo gastroesofágico patológico. O exame clínico oral mostrou: erosões dentárias no grupo 1: 273 faces e no grupo 2: 5; dentes cariados no grupo 1: 23 e 115 no grupo 2; abrasão no grupo 1: 58 e no grupo 2: 95; desgaste por atrito: 408 no grupo 1 e 224 no grupo 2. A face dental mais acometida foi a palatina. No grupo 1, 21 pacientes referiam queixas de aftas freqüentes, 35 sensibilidade dentária, 26 ardência bucal e 42 gosto azedo na boca. Naqueles do grupo 2 estas queixas foram observadas em menor número de pacientes. Conclusĩes - Os doentes com doença do refluxo gastroesofágico apresentam maior incidência de erosões dentárias, aftas, ardência bucal, sensibilidade dentária e gosto azedo que os controles e menor incidência de lesões cariosas em relação aos controles.
\end{abstract}

DESCRITORES - Refluxo gastroesofágico, complicações. Boca. Erosão de dente.

\section{INTRODUÇÃO}

Considerada afecção digestiva de elevada prevalência e incidência em ascensão, a doença do refluxo gastroesofágico (DRGE) compromete a qualidade de vida dos pacientes, em virtude da gravidade das complicações, intensidade e diversidade dos sintomas ${ }^{(9,16,20,25,26,28,31)}$.

O diagnóstico clínico da DRGE é bastante sensível, pois a maioria dos pacientes apresenta os sintomas clássicos da doença, representados por pirose e regurgitação. Todavia, outros sintomas podem ser referidos pelos refluidores, tais como rouquidão, pigarro, tosse crônica, crises de asma e precordialgias, classificados como atípicos. Tais sintomas podem se apresentar associados aos anteriores ou isoladamente ${ }^{(10,17,19,30,34,37) \text {. }}$

Recentemente vários autores têm sugerido que os doentes com DRGE podem apresentar, além dos sintomas citados, manifestações bucais, pois o refluxo, ao atingir a cavidade oral, pode causar lesões nos tecidos moles (aftas) ou mesmo nos dentes, levando a desmineralização, fenômeno conhecido por perimólise ${ }^{(2,5,6,7,15,21)}$.
Os objetivos da presente pesquisa foram analisar as alterações na cavidade oral de pacientes com a DRGE e compará-las com as observadas em indivíduos controles.

\section{MÉTODOS}

Foram avaliados 100 pacientes, 41 homens e 59 mulheres, com idades variando entre 17 e 75 anos (média de 35,5 $\pm 12,9$ ), sendo classificados em dois grupos de 50, sendo o grupo 1 constituído de indivíduos com sintomas de DRGE e o 2 (controle), de indivíduos sem tais queixas. Critérios de inclusão: pacientes de ambos os gêneros, com idade superior a 20 anos e com arcada dentária em bom estado de conservação, com no mínimo 20 dentes. Critérios de exclusão: pacientes com idade inferior a 20 anos, com prótese dentária ou com número de dentes inferior a 20.

Todos foram submetidos a exame clínico da cavidade oral, realizado por um único observador, e responderam a um questionário específico.

Trabalho realizado no Departamento de Cirurgia e Ortopedia da Faculdade de Medicina de Botucatu, Universidade Estadual Paulista - UNESP, Botucatu, SP.

${ }^{1}$ Curso de Pós-graduação em Bases Gerais da Cirurgia; ${ }^{2}$ Departamento de Cirurgia e Ortopedia da Faculdade de Medicina de Botucatu - Universidade Estadual Paulista - UNESP, Botucatu, SP.

Correspondência: Dra. Maria Carolina C. S. F. Corrêa - Rua Eduardo Vergueiro de Lorena, 2-36 - Jardim Aeroporto - 17012-450 - Bauru, SP.

Email: mcarolcorrea@hotmail.com 
Os pacientes do grupo 1 foram submetidos a endoscopia, manometria e pHmetria esofágica prolongada. Tais exames não foram realizados naqueles do grupo 2 , por questões éticas.

Após jejum de 12 horas, os pacientes foram avaliados endoscopicamente, usando o videoendoscópio (GIF - Olympus), que permitiu verificar a presença de hérnia hiatal e/ou as alterações da mucosa esofágica, utilizando-se a classificação proposta por Savary-Miller modificada ${ }^{(35)}$.

Para a realização da manometria esofágica foram utilizados sonda de oito canais, bomba de infusão microcapilar, fisiógrafo de oito canais (Synetics) e microcomputador. A sonda foi introduzida pela narina até o estômago, sendo a seguir tracionada de $1 \mathrm{em} 1$ $\mathrm{cm}$, aguardando-se a estabilização do registro gráfico ${ }^{(29)}$. A análise dos registros gráficos permitiu a obtenção da medida da amplitude da pressão nos esfíncteres inferior e superior do esôfago ( $\mathrm{mm} \mathrm{Hg}$ ), bem como da atividade motora do corpo esofágico.

A pHmetria esofágica de 24 horas foi realizada após suspensão de medicamentos que interferem na secreção ácida por uma semana e jejum de 12 horas, utilizando cateter com sensor de $\mathrm{pH}$ de antimônio e eletrodo de referência externa. O exame foi realizado em ambiente de ambulatório e o processamento dos dados foi efetuado com auxílio de um software, que forneceu vários parâmetros, sendo escolhidos para essa pesquisa a percentagem de tempo nas 24 horas em que o $\mathrm{pH}$ assumia valores abaixo de quatro unidades e o escore de De MEESTER et al. ${ }^{(12,23)}$.

O exame clínico da cavidade oral foi realizado após adequada higiene bucal, onde foram diagnosticadas as lesões cariosas, abrasão, desgaste por atrito e erosões dentárias, segundo a classificação proposta por ECCLES e JENKINS ${ }^{(13)}$.

Para as variáveis que apresentaram distribuição normal foi utilizado teste $t$ de Student, caso contrário, o teste não-paramétrico de Mann-Whitney ${ }^{(11)}$. Para estudo da associação entre as variáveis foi utilizado o teste do qui ao quadrado. Considerou-se o nível de significância de $5 \%$.

\section{RESULTADOS}

Com relação às características demográficas, observou-se que a proporção de indivíduos do sexo feminino no grupo 1 foi de $64 \%$, superior ao do sexo masculino $(P<0,05)$. O grupo 2 (controle) era composto de 23 homens e 27 mulheres $(P>0,05)$. A idade média dos pacientes do grupo $1(36,8 \pm 11,2)$ não diferiu da observada dos controles $(36,3 \pm 14,5)(P>0,05)$.

A endoscopia digestiva alta (EDA) demonstrou hérnia hiatal por deslizamento (HHD) em $76 \%$ dos pacientes com sintomas da DRGE. Em $40 \%$ dos pacientes a EDA permitiu a visualização de erosões (esofagite de refluxo erosiva), que era de leve intensidade na maioria deles (graus I e II). Em 60\% dos pacientes deste grupo não se detectou qualquer lesão na mucosa esofágica (esofagite de refluxo não erosiva - Tabela 1 ).

A pressão no esfíncter inferior do esôfago (EIE) dos pacientes com sintomas de DRGE apresentou valores entre 2,1 e 20,4 $\mathrm{mm} \mathrm{Hg}$ (média de 11,1 $\pm 4,8 \mathrm{~mm} \mathrm{Hg}$ ). Hipotonia esfincteriana (pressão no EIE menor que $10 \mathrm{~mm} \mathrm{Hg}$ ) foi observada em 24 pacientes (48\%). No esfíncter superior do esôfago (ESE) foram detectados valores pressóricos que variaram entre 28,6 e 153,6 $\mathrm{mm} \mathrm{Hg}$, sendo a média de $75 \pm 26,5 \mathrm{~mm} \mathrm{Hg}$ (Tabela 2).
TABELA 1. Achados endoscópicos observados nos pacientes do grupo 1 (classificação de Savary-Miller modificada)

\begin{tabular}{lc}
\hline Achado & $\mathbf{n}(\%)$ \\
\hline ER não erosiva & $30(60 \%)$ \\
ER grau 1 & $14(28 \%)$ \\
ER grau 2 & $1(2 \%)$ \\
ER grau 3 & $2(4 \%)$ \\
ER grau 4 & $0(0 \%)$ \\
ER grau 5 & $3(6 \%)$ \\
Hérnia hiatal & $38(76 \%)$ \\
\hline
\end{tabular}

$E R=$ esofagite de refluxo

TABELA 2. Média, desvio-padrão e intervalo de 95\% de confiança (IC) para as variáveis de amplitude da pressão nos esfíncteres inferior e superior do esôfago (grupo 1- $\mathrm{mm} \mathrm{Hg}$ )

\begin{tabular}{lcc}
\hline Variável & Média e desvio padrão & IC \\
\hline Pressão no EIE & $11 \pm 4,8$ & {$[9,5 ; 12,7]$} \\
Pressão no ESE & $75 \pm 26,5$ & {$[66,3 ; 83,6]$} \\
\hline
\end{tabular}

EIE $=$ esfíncter inferior do esôfago

ESSE $=$ esfíncter superior do esôfago

A pHmetria esofágica de 24 horas foi realizada apenas nos pacientes do grupo 1 por motivos já mencionados. Durante as 24 horas de estudo, a percentagem do tempo em que o $\mathrm{pH}$ esofágico apresentou valores abaixo de quatro unidades (episódio de refluxo gastroesofágico), variou entre 0,6 e $65,56 \%$, sendo a média de $16,9 \pm 13,7$ (Tabela 3). Em 42 pacientes (84\%) os valores observados foram indicativos de refluxo gastroesofágico patológico (maior que $4 \%)^{(12)}$.

TABELA 3. Média, desvio-padrão e intervalo de 95\% (IC) de confiança para as variáveis de pHmetria para o grupo 1

\begin{tabular}{lcc}
\hline Variável & Média e desvio padrão & IC \\
\hline$\%$ 24h com pH esofágico $<4$ & $16,9 \pm 13,7$ & {$[12,4 ; 21,3]$} \\
escore DeMeester & $57,2 \pm 13,7$ & {$[44,3 ; 70,1]$} \\
\hline IC: intervalo de confiança & &
\end{tabular}

Quanto ao escore de De MEESTER et al. ${ }^{(11)}$, os valores extremos observados foram 4,16 e 164,91, com média de 57,2 $\pm 13,7$ (Tabela 3). Escore de De Meester patológico (maior que 14,7) foi observado em $84 \%$ dos pacientes do grupo 1 .

O exame clínico oral detectou várias lesões, sendo erosões, cáries, abrasão e desgastes por atrito as mais comuns (Tabela 4).

TABELA 4. Exame clínico oral: alterações observadas nos 2 grupos

\begin{tabular}{lccc}
\hline Alteração & Grupo 1 & Grupo 2 & Estatística* $^{*}$ \\
\hline Erosão grau 1 & 168 & 5 & $P<0,01$ \\
Erosão grau 2 & 78 & 0 & $P<0,01$ \\
Erosão grau 3 & 27 & 0 & $P>0,05$ \\
$\mathrm{~N}^{\circ}$ total de erosões & 273 & 5 & $P<0,01$ \\
$\mathrm{~N}^{\circ}$ de dentes cariados & 23 & 115 & $P<0,01$ \\
Abrasão & 58 & 95 & $P>0,05$ \\
Desgaste por atrito & 408 & 224 & $P>0,05$ \\
\hline
\end{tabular}

* Teste do Qui ao quadrado

A análise estatística demonstrou que, com relação às erosões de graus 1 e 2 e ao número total de erosões, o grupo 1 superou o grupo 2 (Tabela 4). Com relação às lesões cariosas, o grupo 2 apresentou maior incidência que o grupo $1(P<0,01)$. 
Outro aspecto analisado ao exame clínico oral foi o número de faces dentárias acometidas pela erosão. A análise estatística demonstrou que quanto maior o número de erosões, maior era o acometimento da face palatina (Figura 1).

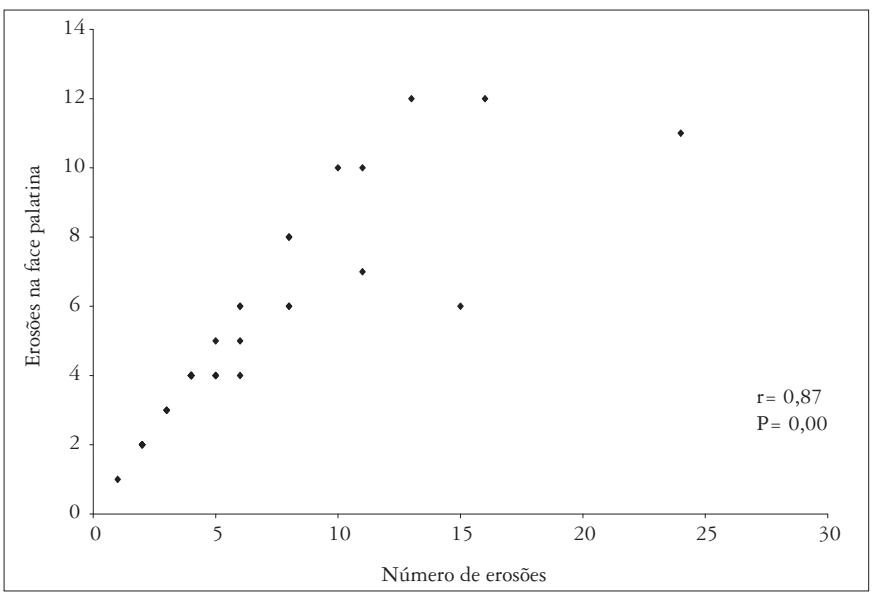

FIGURA 1. Diagrama de dispersão relativo ao número total de erosões e o observado na face palatina

Todos os pacientes responderam ao questionário específico, em que foram analisados alguns sintomas que poderiam estar relacionados ao refluxo ácido para a cavidade oral. As respostas afirmativas encontram-se na Tabela 5. A análise estatística pelo teste do qui ao quadrado (Figura 2) demonstrou que os pacientes com sintomas da DRGE referiam percentuais mais elevados que os controles com relação à presença de aftas, sensibilidade dentária, ardência bucal e gosto azedo.

TABELA 5. Análise dos sintomas relacionados ao refluxo ácido para a cavidade oral (número de afirmativas)

\begin{tabular}{lccc}
\hline Sintomas & Grupo 1 & Grupo 2 & Estatística* $^{*}$ \\
\hline Aftas freqüentes & $21(42 \%)$ & $2(4,0 \%)$ & $\mathrm{P}<0,001$ \\
Sensibilidade dentária & $35(70 \%)$ & $13(26 \%)$ & $\mathrm{P}<0,001$ \\
Ardência bucal & $26(52 \%)$ & $1(2,0 \%)$ & $\mathrm{P}<0,001$ \\
Gosto azedo & $42(89 \%)$ & $3(6,0 \%)$ & $\mathrm{P}<0,001$ \\
\hline * Teste do Qui ao quadrado & & &
\end{tabular}

* Teste do Qui ao quadrado

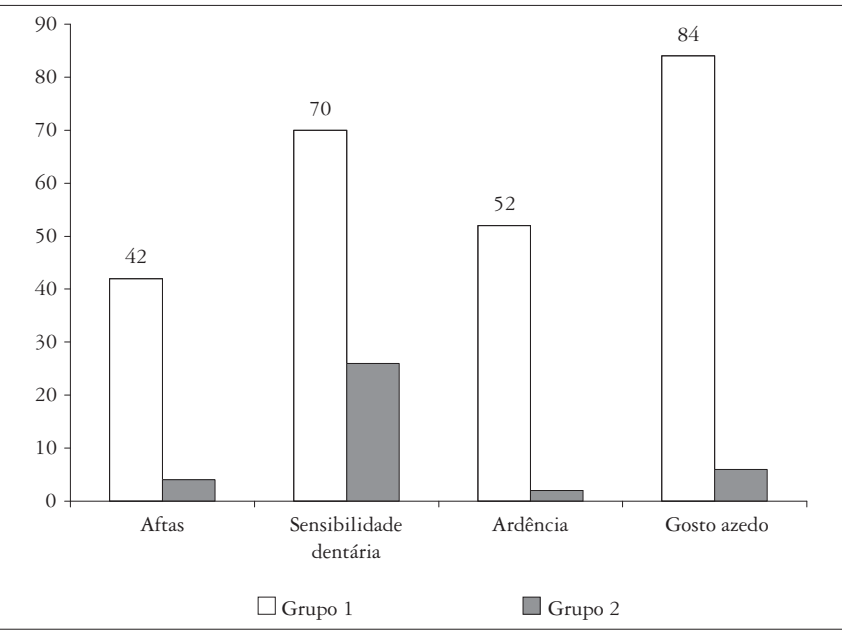

FIGURA 2. Percentual de indivíduos com aftas, sensibilidade dentária, ardência e gosto azedo nos dois grupos de estudo

\section{DISCUSSÃO}

Para o estudo das alterações da cavidade oral em pacientes com a DRGE optou-se pela pesquisa prospectiva, considerada na literatura como padrão-ouro de pesquisa em saúde ${ }^{(1)}$

Neste estudo foi observado que no grupo 1 (DRGE), 64\% dos pacientes pertenciam ao sexo feminino. A maior incidência da DRGE em mulheres tem sido referida por alguns autores ${ }^{(31}$, 32). Em extenso estudo populacional realizado em Pelotas, RS, OLIVEIRA et al. ${ }^{(31)}$, observaram que a DRGE apresenta maior prevalência entre as mulheres, em geral associada a eventos estressantes negativos e mal estar psicológico.

A idade dos pacientes do grupo $1(36,8 \pm 11,2)$ não diferiu da observação no grupo controle $(36,3 \pm 14,5 P>0,05)$, mostrando que os grupos eram homogêneos. A idade média dos pacientes com DRGE $(36,8 \pm 11,2)$ foi semelhante à referida por SOMANI et al. ${ }^{(36)}$, porém inferior à publicada por outros autores ${ }^{(3,25,27,32)}$.

O diagnóstico de HHD foi realizado em 76\% do pacientes do grupo 1, resultado semelhante ao observado por LOFFELD e PUTTEN ${ }^{(26)}$. A HHD tem sido considerada como importante fator patogênico da DRGE. Alguns autores ${ }^{(1,4)}$ demonstraram que em pacientes com HHD volumosa (maiores que $5 \mathrm{~cm}$ ), a percentagem de refluxo ácido e a intensidade da esofagite são mais elevadas que naqueles com hérnia não-volumosa $(P<0,05)$.

Em estudo epidemiológico realizado na Holanda, LOFFELD e PUTTEN ${ }^{(26)}$ observaram que nos últimos 10 anos houve aumento do número de pacientes com esofagite de refluxo gastroesofágico em graus 1 e 2 . Coincidentemente, foi observado nesta série que dos 20 pacientes com esofagite erosiva, 15 apresentaramna em graus leve e moderado ( 1 e 2 ). A explicação para esse fato se deve ao crescente interesse dos médicos pela doença em questão, encaminhando os pacientes precocemente para avaliação endoscópica. O esôfago de Barrett foi diagnosticado em 6\% desta casuística, índice inferior ao publicado por LOPES et al. ${ }^{(27)}$ e PAULA ${ }^{(33)}$, que referiram taxas de $35,6 \%$ e $25 \%$, respectivamente.

O valor médio da pressão no EIE dos pacientes do grupo 1 foi de $11 \pm 4,8 \mathrm{~mm} \mathrm{Hg}$, semelhante ao publicado por PASTORE et al. ${ }^{(32)}$, porém mais elevado que o observado por vários autores, que referiram níveis pressóricos variando entre 4,8 $\mathrm{mm} \mathrm{Hg}$ e $9 \mathrm{~mm} \mathrm{Hg}^{(14,27)}$. A hipotonia esfincteriana (EIE) foi observada em $48 \%$ dos pacientes, índice que coincide com o referido por $\mathrm{NASI}^{(30)}$.

Com relação ao ESE, o valor médio observado nos pacientes do grupo 1 foi de $75 \pm 26,5 \mathrm{~mm} \mathrm{Hg}$, mais elevado que o publicado por LEMME et al. ${ }^{(24)}$. O resultado encontrado no presente estudo causou surpresa, pois esperava-se valor mais baixo. Nesta eventualidade, o conteúdo refluído chegaria até a cavidade oral com mais facilidade. Em estudo realizado em 251 indivíduos com DRGE e sintomas típicos, $\mathrm{CORSI}^{(8)}$ observou valor médio da pressão no ESE de $62,7 \pm 37,2 \mathrm{~mm} \mathrm{Hg}$.

A pHmetria prolongada demonstrou refluxo anormal em $84 \%$ dos pacientes do grupo 1, índice semelhante ao publicado por LEMME et al. ${ }^{(23)}$. Em oito pacientes $(16 \%)$ a percentagem do tempo com o refluxo ácido esteve abaixo de $4 \%$, isto é, dentro da normalidade, resultado inferior ao referido por $\mathrm{CORSI}^{(8)}$. Tal 
discrepância pode ser explicada pela metodologia empregada. Enquanto que na presente série a $\mathrm{pHmetria}$ esofágica foi realizada em ambiente de ambulatório, no trabalho de $\mathrm{CORSI}^{(8)}$, ela se fez em ambiente hospitalar.

O exame clínico oral foi realizado nos pacientes dos dois grupos, demonstrando maior incidência de erosões dentárias nos doentes com DRGE que nos controles $(P<0,001$ - Tabela 4), resultado semelhante ao observado por vários autores ${ }^{(2,5,7,15,22)}$.

LAZARCHIK e FILLER ${ }^{(22)}$ referem que as erosões decorrentes da ingestão crônica de ácido localizam-se na superfície vestibular dos dentes anteriores. Todavia, nas erosões de causa intrínseca (DRGE e distúrbios alimentares), o processo de descalcificação envolve as superfícies lingual e palatal dos dentes anteriores e oclusal dos dentes posteriores ${ }^{(6,18)}$. Neste estudo as erosões estavam localizadas em maior número nas superfícies lingual e palatal dos dentes anteriores, em concordância com os autores citados.

Com relação às lesões cariosas, foi observado que nos doentes com DRGE o número de cáries era inferior ao encontrado nos controles $(P<0,001$ - Tabela 4$)$. Este resultado causou surpresa, pois pensava-se que a DRGE poderia favorecer o aparecimento de lesões cariosas, uma vez que a cárie necessita de $\mathrm{pH}$ ácido para se desenvolver.

Os resultados dessa pesquisa com respeito às cáries, estão em concordância com o publicado por CAZZONATO Jr. et al. ${ }^{(7)}$, que referem a relação inversa entre cáries e refluxo gastroesofágico.

A etiologia da lesão cariosa é multifatorial, resultando do desequilíbrio entre fatores extrínsecos (microorganismos da placa bacteriana) e intrínsecos (hospedeiro). Os microorganismos envolvidos na cariogênese são estreptococos, lactobacilos, enterococos e actinomicetos.
Em raciocínio simplista, poder-se-ia supor que o material refluído com baixo $\mathrm{pH}$ destruiria os microorganismos da placa bacteriana, levando à redução das cáries nos pacientes com DRGE. Entretanto, tal explicação não tem respaldo científico, pois as bactérias acima listadas são ácido-resistentes ${ }^{(7)}$. Outras hipóteses aventadas seriam que o ácido deixaria a superfície dos dentes lisas e polidas, impedindo o acúmulo de placa bacteriana ou ainda que os indivíduos com DRGE realizariam sua higiene oral maior número de vezes, devido ao gosto azedo provocado pelo refluxo ácido.

Além do fator bacteriano, outros relacionados ao hospedeiro são também importantes na fisiopatologia de lesão cariosa, todos relacionados à saliva, tais como o fluxo, a capacidade tampão e a concentração de eletrólitos e imunoglobulinas. Assim, novas pesquisas analisando esses fatores devem ser realizadas para melhor compreensão das alterações bucais observadas nos pacientes com DRGE.

\section{CONCLUSÕES}

Os resultados da presente pesquisa permitem concluir que os pacientes com DRGE apresentam:

a) maior incidência de erosões dentárias, aftas, ardência bucal, sensibilidade dentária e gosto azedo que os controles;

b) número reduzido de lesões cariosas em relação aos controles.

\section{AGRADECIMENTOS}

Os autores agradecem à Fundunesp (Fundação para o desenvolvimento da UNESP) pelo auxílio recebido, à Capes pela bolsa de estudos à primeira autora e à empresa Dynamed, pelo fornecimento de $\mathrm{pH}$ metro.

Corrêa MCCSF, Lerco MM, Henry MACA. Study in oral cavity alterations in patients with gastroesophageal reflux disease. Arq Gastroenterol. 2008;45(2):132-6.

ABSTRACT - Background - The gastroesophageal reflux disease, which has become highly and increasingly incident, may be manifested by typical (pyrosis and regurgitation) and atypical (pulmonary, otorhinolaryngological and buccal) symptoms. Aim - To analyze alterations in the oral cavity patients with gastroesophageal reflux disease. Methods - One hundred patients were studied being 50 gastroesophageal reflux disease patients (group 1) and 50 controls (group 2). All patients were submitted to an oral clinical exam and specific survey. Patients in group 1 were submitted to upper endoscopy, manometry and esophageal pH monitoring. Results - The upper endoscopy revealed esophagitis in all patients, 20 erosive esophagitis, 30 no-erosive esophagitis and 38 hiatal hernia. Average pressure of the lower esophageal sphincter was $11 \pm 4,8 \mathrm{~mm} \mathrm{Hg}$ and of the upper esophageal sphincter $75 \pm 26,5 \mathrm{~mm} \mathrm{Hg}$. In $42 \mathrm{patients}$ of group 1 (84\%) pathological gastroesophageal reflux was observed. Clinical exams revealed: dental erosions in group 1: 273 faces and in group 2: 5 tooth decays in group 1: 23 and 115 in group 2; abrasion in group 1: 58 and in group 2: 95; attrition wear: 408 in group 1 and 224 in group 2. The most damages was the palatine face. In group 1, 21 patients complained about frequent episodes of cankers sores, 35 of tooth sensibility, 26 of burning mouth and 42 of sour taste in the mouth. In group 2 the complaints were observed in lower number of patients. Conclusions - Patients with gastroesophageal reflux disease present higher incidence of dental erosion, cankers sores, mouth burning sensation, sensitivity and sour taste than controls. Patients with gastroesophageal reflux disease show lower incidence of tooth decays as compared to controls.

HEADINGS - Gastroesophageal reflux, complications. Mouth. Tooth erosion. 


\section{REFERÊNCIAS}

1. Abrahão Jr LJ, Lemme EM, Carvalho BB, Alvariz A, Aguero GC, Schechter RB. Relação entre o tamanho de hérnia hiatal e tempo de exposição ácida esofágica nas doenças do refluxo erosiva e não-erosiva. Arq Gastroenterol. 2006;43:37-40.

2. Ali DA, Brown RS, Rodriguez LO, Moody EL, Nasr MF. Dental erosion caused by silent gastroesophageal reflux disease. J Am Dent Assoc. 2002;133:734-7.

3. Aranha NC, Brandalise NA. Tratamento videolaparoscópico da esofagite de refluxo pela técnica "mista" (Nissen modificada). GED Gastroenterol Endosc Dig. 1995;14:219-22.

4. Balbinotti SS, Soldera J, Pilla PC, Bernardi LS, Balbinotti RA. Relação entre o tamanho da hérnia hiatal e a gravidade da esofagite de refluxo. GED Gastroenterol Endosc Dig. 2007;28:147-50.

5. Barron RP, Carmichael RP, Marcon MA, Sàndor GK. Dental erosion in gastroesophageal reflux disease. J Can Dent Assoc. 2003;69:84-9.

6. Bartlett DW, Evans DF, Anggiansah A, Smith BG. A study of association between gastro-oesophageal reflux and palatal dental erosion. Br Dental J. 1996;181:12531.

7. Cazzonato Jr H, Bernasconi GCR, Pedrazzolli JR. Gastroesophageal reflux and oral lesions: is the acid that bad? GED Gastroenterol Endosc Dig. 2003;22:42-6.

8. Corsi PR. Fatores clínicos e de diagnóstico do padrão e da intensidade do refluxo nos pacientes com sintomas típicos da doença do refluxo gastroesofágico [tese]. São Paulo: Faculdade de Ciências Médicas da Santa Casa de São Paulo; 2006

9. Cuenca-Abente F, Faerberg A, Marty PF, Corti R. Sintomas respiratorios asociados a la enfermedad por reflujo gastroesofágico: diagnóstico y tratamiento. Acta Gastroenterol Latinoam. 2006;36:42-50

10. Curi PR. Metodologia e análise da pesquisa em ciências biológicas. Botucatu: Tipomic, 1997. p.32-3

11. DeMeester TR, Wang CI, Wernly JA, Klementschitsch P, Bermudez G, Johnson LF, Skinner DB. Technique, indications, and clinical use of 24 hour esophageal pH monitoring. J Thorac Cardiovasc Surg. 1980;79:656-70.

12. DeMeester TR, Bonavina L, Iascone C, Courtney JV, Skinner DB. Chronic respiratory symptoms and occult gastroesophageal reflux. A prospective clinical study and results of surgical therapy. Ann Surg. 1990;211:337-45.

13. Eccles JD, Jenkins WG. Dental erosion and diet. J Dent. 1974;2:153-9.

14. Felix VN, Yogi I, Perini M, Echeverria R, Bernardi C. Surgical treatment of the noncomplicated gastroesophageal reflux: fundoplication without division of the short gastric vessels. Arq Gastroenterol. 2002;39:93-7.

15. Gregory-Head BL, Curtis DA, Kim L, Cello J. Evaluation of dental erosion in patients with gastroesophageal reflux disease. J Prosthet Dent. 2000;83:675-80.

16. Gurski RR, da Rosa AR, Valle E, de Borba MA, Valiati AA. Manifestações extra-esofágicas da doença do refluxo gastroesofágico. J Bras Pneumol. 2006;32:150-60.

17. Harding SM, Sontag SJ. Asthma and gastroesophageal reflux. Am J Gastroenterol 2000;95(Supp1 8):s523-s32.

18. House RC, Grisius R, Bliziotes MM, Licht JH. Perimolysis: unveiling the surreptitious vomiter. Oral Surg Oral Med Oral Pathol. 1981;51:152-5.

19. Koufman JA, Amin MR, Panetti M. Prevalece of reflux in 113 consecutive patients with laryngeal and voice disorders. Otolaryngol Head Neck Surg. 2000;123: $385-8$.
20. Kulig M, Leodolter A, Vieth M, Schulte E, Jaspersen D, Labenz J, Lind T, MeyerSabelle KW, Malfertheiner P. Quality of life in relation to symptoms in patients with gastro-oesophageal reflux disease - an analysis based on the ProGERD initiative. Aliment Pharmacol Ther. 2003;18:767-76.

21. Lazarchik DA, Filler SJ. Effects of gastroesophageal reflux on the oral cavity. Am J Med. 1997;103:107s-13s

22. Lazarchik DA, Filler SJ. Dental erosion: predominant oral lesion in gastroesophageal reflux disease. Am J Gastroenterol. 2000;95(8 Suppl):s33-s8.

23. Lemme EM, Almeida SM, Firman CM, Pantoja JP, Nascimento FA. pH metria esofagiana prolongada - avaliação de 170 exames. Arq Gastroenterol. 1997;34:71-7.

24. Lemme EM, Domingues GR, Silva LF, Firman CG, Pantoja JP. Esofagomanometria computadorizada: resultados preliminares em voluntários adultos saudáveis. GED Gastroenterol Endosc Dig. 2001;20:29-35.

25. Liano AD, Yárnoz C, Artieda C, Flores L, Garde C, Romeo I, Ortiz H. Gastroesophageal reflux: prevalence of psychopathological disorders and quality of life implications. Dis Esophagus. 2006;19:373-6.

26. Loffeld RJ, van der Putten AB. Rising incidence of reflux oesophagitis in patients undergoing upper gastrointestinal endoscopy. Digestion. 2003;68:141-4.

27. Lopes LR, Brandalise NA, Andreollo NA, Leonardi LS. Tratamento cirúrgico videolaparoscópico da doença do refluxo gastroesofagiano: técnica de Nissen modificada - resultados clínicos e funcionais. Rev Assoc Med Bras. 2001;47:141-8.

28. Moraes-Filho JP, Chinzon D, Eisig JN, Hashimoto CL, Zaterka S. Prevalence of heartburn and gastroesophageal reflux disease in the urban Brazilian population. Arq Gastroenterol. 2005;42:122-7.

29. Morais DJ, Lopes LR, Andreollo NA. Manometria esofágica: indicações clínicas GED Gastroenterol Endosc Dig. 2007;26:79-85.

30. Nasi A. Doença do refluxo gastroeofágico: reavaliação clínica endoscópica e da monitorização do pH intraluminar esofágico (tese). São Paulo: Faculdade de Medicina da Universidade de São Paulo; 1996.

31. de Oliveira SS, dos Santos Ida S, Silva JF, Machado EC. Prevalência e fatores associados à doença do refluxo gastroesofágico. Arq Gastroenterol. 2005;42:116-21.

32. Pastore R, Crema E, Silveira MC, Presoto AF, Herbella FA, Del Grande JC. Eletromanometria esofágica e $\mathrm{pH}$ metria de 24 horas na avaliação pós-operatória da hiatoplastia e válvula anti-refluxo total laparoscópica. Arq Gastrenterol. 2006;43:112-6.

33. Paula AL. Fundoplicatura total laparoscópica no tratamento cirúrgico da doença do refluxo gastroesofágico [tese]. São Paulo: Universidade de São Paulo; 1997.

34. Richter JE. Extraesophageal presentations of gastroesophageal reflux disease: an overview. Am J Gastroenterol. 2000;95(Suppl 8):51-3.

35. Savary M, Miller G. The esophagus: handbook and atlas of endoscopy. Solothurn, Schweiz: Gassman; 1978.

36. Somani SK, Ghoshal UC, Saraswat VA, Aggarwal R, Misra A, Naik SR, Krishnani N. Correlation of esophageal $\mathrm{pH}$ and motor abnormalities with endoscopic severity of reflux esophagitis. Dis Esophagus. 2004;17:58-62.

37. Wetscher GJ, Glaser K, Hinder RA, Perdikis G, Klingler P, Bammer T, Wieschemeyer T, Schwas G, Kinger A, Pointner R. Respiratory symptoms in patients with gastroesophageal reflux disease following medical therapy and following anti-reflux surgery. Am J Surg. 1997;174:639-43. 